\title{
A Cell Cycle Model for Somitogenesis: Mathematical Formulation and Numerical Simulation
}

\author{
J. R. Collier,* D. McInerney,† S. Schnell, $\$$ P. K. Maini,,$„ \|$ D. J. Gavaghan, \\ P. Houston** and C. D. STERn††
}

\begin{abstract}
*†\$Centre for Mathematical Biology, Mathematical Institute, 24-29 St Giles', Oxford OX1 3LB, U.K., -Computing Laboratory, Wolfson Building, Parks Road, Oxford OX1 3QD, U.K., **Department of Mathematics and Computer Science, University of Leicester, University Road, Leicester LE1 7RH, U.K. and $\dagger \dagger$ Department of Genetics and Development, Columbia University, $701 \mathrm{~W}$ est 168th Street, New York,
\end{abstract} NY 10032, U.S.A.

(Received on 14 March 2000, Accepted in revised form on 11 August 2000)

\begin{abstract}
After many years of research, the mechanisms that generate a periodic pattern of repeated elements (somites) along the length of the embryonic body axis is still one of the major unresolved problems in developmental biology. Here we present a mathematical formulation of the cell cycle model for somitogenesis proposed in Development 105 (1989), 119-130. Somite precursor cells in the node are asynchronous, and therefore, as a population, generate continuously pre-somite cells which enter the segmental plate. The model makes the hypothesis that there exists a time window within the cell cycle, making up one-seventh of the cycle, which gates the pre-somite cells so that they make somites discretely, seven per cycle. We show that the model can indeed account for the spatiotemporal patterning of somite formation during normal development as well as the periodic abnormalities produced by heat shock treatment. We also relate the model to recent molecular data on the process of somite formation.
\end{abstract}

(C) 2000 Academic Press

\section{Introduction}

Most metazoan animals have some segmental organization of their body axis. In vertebrate embryos, segmentation occurs along the craniocaudal (head-tail) axis, and is first seen clearly when somites form as spheres of cells on each side of the axis, in an orderly anterior-posterior (AP) sequence (for a review, see Gossler \& Hrabě de Angelis, 1998).

The initial segmental pattern of the somites is important because it governs the segmental or-

$\|$ Author to whom correspondence should be addressed. E-mail:maini@maths.ox.ac.uk ganization of peripheral spinal nerves, vertebrae, axial muscles, and the metameric distribution of early blood vessels. Genetic or environmental factors sometimes disturb somitogenesis, and there are many clinical conditions which occur as a result (Zhang \& Gridley, 1998; Evrard et al., 1998).

In the past four decades, our knowledge of the mechanisms of somitogenesis has increased considerably. The formation and differentiation of somites appears to result from three distinct morphological events progressing in a strict temporal-spatial order: (1) pre-patterning of the pre-somitic mesoderm (PSM); (2) somite and 
somite boundary formation; and (3) the differentiation of each somite into anterior and posterior halves and subsequently into dorsal (dermomyotome) and ventral (sclerotome) domains (Keynes \& Stern, 1988; Gossler \& Hrabě de Angelis, 1998).

The pre-patterning of somites appears to be established before segmentation. Scanning electron microscopy observations suggest metameric arrangements of groups of cells, named somitomeres (see, for a review, Jacobson $\&$ Meier, 1986). The existence of a pre-pattern is consistent with the finding that reversal of the AP axis of the PSM leads to reversed somites (see, for a review, Keynes \& Stern, 1988).

There is very strong evidence that somite and somitic boundary formation is controlled by a segmentation clock. The first experimental evidence to support the segmentation clock was obtained after heat shock was applied to amphibian embryos (Elsdale et al., 1976; Cooke, 1978; Pearson \& Elsdale, 1979; Veini \& Bellairs, 1986). These generate a discrete segmentation abnormality, which appears after a fixed number of somites formed following the heat shock. When a single heat shock is applied to chick embryos (Primmett et al., 1988) several somite anomalies, which may be uni- or bilateral, appear separated by relatively constant distances of six to seven normal somites. The repeated anomalies suggested that heat shock affects an oscillatory process within the somite precursors (Primmett et al., 1988, 1989; Stern et al., 1988). In addition, there appears to be some degree of cell cycle synchrony between the cells in the PSM which are designed to segment together to form a somite (Stern \& Bellairs, 1984). Similar periodic anomalies in somite formation can also be caused by drugs inhibiting cell cycle progression (Primmett et al., 1989). These experimental observations link the segmentation clock with the cell cycle.

Recently, the study of the expression of $c$ hairy-l and lunatic fringe (l-fng) in the PSM of chick embryos has provided molecular evidence for the existence of a segmentation clock (Palmeirim et al., 1997; McGrew et al., 1998). During segmentation, the cells of the PSM go through at least 12 cycles of $c$-hairy- 1 expression before becoming part of a somite, while more cells are continuously incorporated into the posterior end of the PSM. Based on this observation, it was proposed that the segmentation clock controls the time duration of cells in the PSM before they form part of a somite (Pourquié, 1999; Schnell $\&$ Maini, 2000). During the time taken for one somite to form, the expression of c-hairy-1 sweeps along the PSM in the posterior-anterior direction, narrowing as it moves along. This wavefront-like expression finally stops and is maintained only within the caudal half of each forming somite. The $c$-hairy- 1 expression is independent of cell movements; it is an intrinsic cell autonomous property of this tissue (Palmeirim et al., 1997; McGrew \& Pourquié, 1998; Pourquié, 1998). More recently, McGrew et al. (1998) and Forsberg et al. (1998) have shown that l-fng gene expression resembles the expression of c-hairy-1 in PSM. In fact, both expressions are coincident and appear to be regulated by the same segmentation clock.

There is a change in the mechanical properties of the cells in the PSM before they differentiate into a somite. There is an increase in cell compaction, cell-cell adhesion and in the expression of $\mathrm{N}$-cadherin, which is followed by epithelialization (Keynes \& Stern, 1988; Tam \& Trainor, 1994) into somites. Additionally, the cells become polarized around a central lumen (somitocoele).

Although much is known about somitogenesis, its mechanism is still one of the major unresolved problems in developmental biology. During the last three decades, several models have been proposed to explain the formation of somites: the clock and wavefront model (Cooke \& Zeeman, 1976), the wave gradient model (Wilby \& Ede, 1975; Flint et al., 1978), a reaction-diffusion-type model (Meinhardt, 1982, 1986), the cell cycle model (Stern et al., 1988; Primmett et al., 1989), the wave and cell polarization model (Polezhaev, 1992, 1995a, b) and a clock and induction model (Schnell \& Maini, 2000). Some of these successfully account for a number of aspects of somitogenesis, but fail to explain, or even contradict other observations (see, for a review Schnell et al., 2000).

The cell cycle model incorporates several known aspects of somitogenesis better than most models; for example, it can account for the periodic abnormalities observed after a single heat shock, the cell synchrony along the PSM, the change in the mechanical properties of the cells in 
the PSM before they differentiate into a somite, as well as the cell autonomous character of somite formation in PSM explants and the transplantation experiments reversing the AP axis.

Criticisms have been made of the cell cycle model. Palmeirim et al. (1997) and Roy et al. (1999) argue against a direct role for the cell cycle in somite formation. Firstly, Palmeirim et al. (1997) indicated that the cycling times of c-hairy-1 expression, a product of the segmentation clock, occurs in the chick embryo with a periodicity similar to the time it takes to form a single somite (approximately $90 \mathrm{~min}$ ) rather than the observed cell cycle length $(9 \mathrm{hr})$. However, we do not yet know whether the major role of c-hairy-1 and l-fng is in the allocation of cells to individual somites, which is the subject of the cell cycle model, or rather in the subdivision of somites into rostral and caudal compartments. If the latter is true, then two separate clocks may exist: one with a 9-hr period, coupled to the cell cycle, and one with a 90 -min period, driving the expression of c-hairy-l and l-fing.

Secondly, Roy et al. (1999) suggest that a reevaluation must be made of the relation between the segmentation process and the cell cycle durations in vertebrates after finding that in zebrafish embryos heat shock anomalies occur in periodic units of five somites which do not closely match with the cell cycle duration. However, it is worth noting that the anomalies in zebrafish appear at exactly twice the frequency predicted by the cell cycle model. Together with the finding that this group of teleosts has undergone an additional round of genome duplication (see Richardson et al., 1997), one possible scenario is that two separate genes (and/or two separate clocks linked to the cell cycle, but half-a-cycle out of phase with one another) regulate somitogenesis in these animals. Supporting this suggestion is the finding that another member of the hairy-enhancer of split family, her-1, has been characterized as having an expression pattern in every other somite (Müller et al., 1996), unlike any gene thus far described in other vertebrates.

Therefore, the cell cycle model is still a realistic model that captures many of the experiment observations in most vertebrate systems and, in particular, is the only model to date that can explain the heat shock experiments. Hence, we believe that the model warrants further exploration.

In the present work, we develop a mathematical formulation of the cell cycle model for somitogenesis. After an exposition of the descriptive model, we introduce its assumptions and a mathematical formulation in Section 2. Then we derive parameter constraints under which the model must operate (Section 3). In Section 4, we present a qualitative description of the behaviour of the model and solve the governing equations numerically using a finite element method. In Section 5, we introduce the effects of heat shock into the cell cycle model and show that it exhibits the periodic abnormalities observed experimentally. Finally, we briefly discuss the results of our model in Section 6.

\section{Cell Cycle Model}

The cell cycle model of Stern et al. (1988) and Primmett et al. (1989) links the cell cycle with somite segmentation. This hypothesis is supported by the following observations: (1) the existence of discrete regions of cell synchrony in the PSM, (2) a cell cycle duration of $9 \mathrm{hr}$, which corresponds to the development of six to seven somites in the chick embryo, and (3) periodic anomalies caused by heat shock experiments are mimicked by drugs inhibiting the cell cycle progression.

According to the cell cycle model, cells destined to form somites leave Hensen's node strictly in the order in which they were derived from founder cells in the node, and they remain in that order. Hence, there is some degree of cell cycle synchrony of somite cells at the same level, and these cells will be arranged along the embryonic axis in the same order as they are positioned in their cell cycles; older cells further anterior than younger ones (Stern et al., 1992).

The formation of a somite is explained by assuming that there are two successive time points, $P_{1}$ and $P_{2}$, a time interval of $90 \mathrm{~min}$ apart perhaps within the $M$ phase of the cell cycle, near the time of the second last mitotic division before segmentation. This determination phase comprises about one-seventh of the total cell cycle time. Somite cells recognize the $P_{1}-P_{2}$ time window. Since there is some degree of cell synchrony 


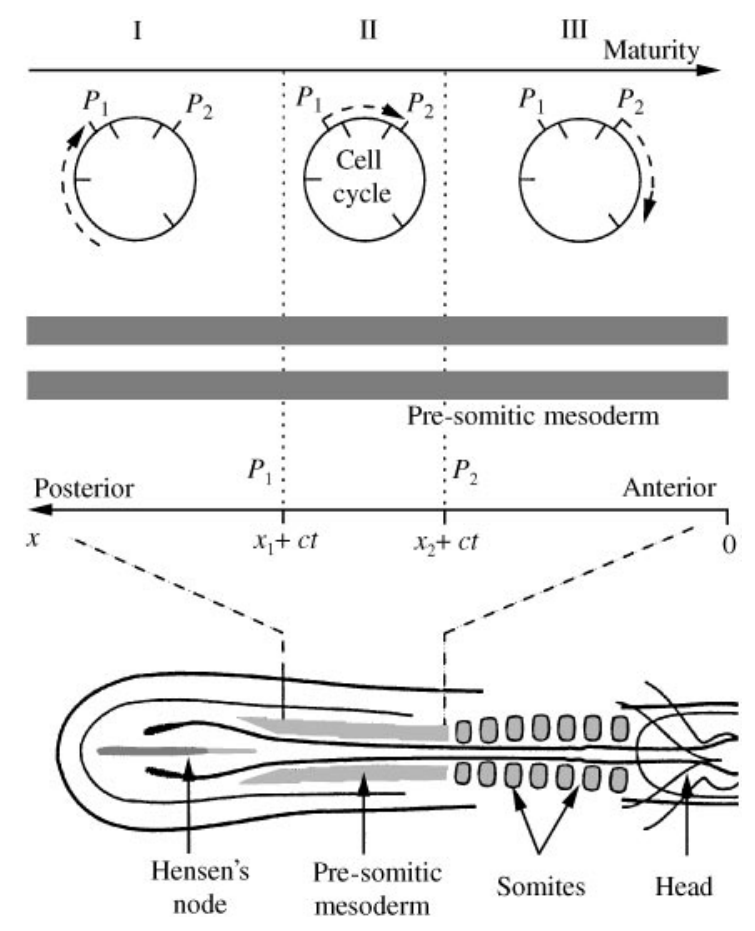

FIG. 1. Diagrammatic representation of the cell cycle model illustrating the two special points $P_{1}$ and $P_{2}$ postulated to exist in the cell cycle, and the three key stages proposed by the model. Cells at the posterior-most margin of the PSM (I) are at a younger developmental age than those undergoing somitogenesis. As these cells mature, they become competent (II) to respond to the signalling molecule which is secreted by cells at $P_{2}$. During this phase cells are triggered to form somites as they mature to the next stage (III). At this stage they are no longer able to respond to or to secrete the signal (refractory stage).

in the cells destined to segment together, some cells will reach $P_{2}$ before others. These cells produce a signal to which cells situated between $P_{1}$ and $P_{2}$ would respond to by later increasing their adhesion to each other shortly before segmentation, regardless of their position within the PSM. The resulting group of cells would actually undergo segmentation one cell cycle after the $P_{1}-P_{2}$ time window. After the $P_{1}-P_{2}$ window, cells become refractory to the signal and/or unable to signal (see Fig. 1).

The model proposes that heat shock temporarily blocks the cell cycle, so altering the number of cells that become adhesive together. Such an alteration would occur once in each cell cycle in the segmental plate posterior to the $P_{1}-P_{2}$ time window, accounting for the repetitive anomalies resulting from heat shock.

\subsection{MATHEMATICAL FORMULATION}

To develop a mathematical formulation of the cell cycle model, we introduce two assumptions:

(i) Cells move up through the PSM, but for mathematical convenience we take axes fixed with respect to the cells so that we can consider the pattern (including the node and the PSM) to be moving down the rostrocaudal axis. We assume that the length of the PSM is constant on average, and that the pattern moves with constant speed $c$ down the embryonic axis, in normal conditions. A consequence of the constant speed of the pattern is that mitosis (and other given points of the cell cycle) occur at positions fixed in the pattern. We assume that the speed of the pattern $c \approx 0.02 \mu \mathrm{ms}^{-1}$. This appears to be reasonable for the trunk region of the chick.

(ii) The signal given by cells reaching $P_{2}$ is like a pulse, the signalling molecule is short-lived and diffuses rapidly. Ideally, only those cells between $P_{1}$ and $P_{2}$ at the time the signal is first given would respond. Rapid diffusion and/or clustering of cells is required to ensure that all cells between $P_{1}$ and $P_{2}$ respond.

To simplify the analysis, we study the model in one space dimension and on one side of the embryo. The latter assumes that there is no signalling between the two parallel axes of PSM. This is biologically reasonable (Keynes \& Stern, 1984; Veini \& Bellairs, 1986). We let $x$ denote the distance in the AP axis, where the origin is taken to be fixed with respect to a given somite, and $t$ denotes time. We assume that the key step in determining a cell's fate as part of a somite is its production of a somitic factor, $G$, and we denote its concentration by $g(x, t)$. This somitic factor might be a transcription factor or a precursor of an adhesion molecule, produced in response to a diffusive signalling molecule $S$, with concentration $s(x, t)$.

In accordance with the assumptions, $G$ is produced only when a cell has passed $P_{1}$ and its production is triggered by the chemical signal $S$. The model equations are

$$
\frac{\partial g}{\partial t}=\frac{(g+\mu s)^{2}}{\gamma+\rho g^{2}} \theta(x, t)-\eta g,
$$




$$
\frac{\partial s}{\partial t}=\frac{\kappa}{\varepsilon+g} \chi(x, t)-\lambda s+D \frac{\partial^{2} s}{\partial x^{2}},
$$

where

$$
\begin{aligned}
& \theta(x, t)=H\left(c t-x+x_{1}\right), \\
& \chi(x, t)=H\left(c t-x+x_{2}\right) .
\end{aligned}
$$

The Heaviside function, $H$, is of the general form $H(\alpha-x)$, where

$$
H(\alpha-x)= \begin{cases}1 & \text { if } x \leqslant \alpha \\ 0 & \text { if } x>\alpha\end{cases}
$$

for any $\alpha$, and $\mu, \gamma, \rho, \eta, \kappa, \varepsilon, \lambda, D, c, x_{1}, x_{2}$ are positive constants, with $x_{2}<x_{1}$. Here $x_{i}$ is the position of $P_{i}$ on the $x$-axis when $t=0$, for $i=1,2$. Thus, $G$ production can only occur in cells anterior to $P_{1}$, and the source term in the $s$ equation (2) is zero until the cells pass $P_{2}$.

One of the crucial assumptions in the cell cycle model is the switch-behaviour occurring in the kinetics of the somitic factor $G$. This is captured in the above mathematical formulation by the right-hand side of eqn (1). Such kinetics could arise from linear degradation of $G$ (the $-\eta g$ term) and production of $G$ via the term $(g+\mu s)^{2} /\left(\gamma+\rho g^{2}\right)$ which represents the autocatalysis of $G$ and enhanced $G$ production by $S$, with saturation for large $g$. The term $\kappa /(\varepsilon+g)$ in eqn (2) assumes that $S$ is inhibited by $G$, so that cells which have already been specified as somitic (and hence have a high level of $g$ ) cannot signal. We assume that $S$ also degrades linearly (the $-\lambda s$ term) and diffuses rapidly.

The embryonic axis lengthens, and the node and segmental plates move down it at rate $c$, so the spatial domain is stationary. To be specific, we consider

$$
0 \leqslant x \leqslant d(t), \quad t \geqslant 0
$$

where $d(t)$ is the position of a point which moves down the axis with the pattern, at speed $c$. For points on the axis posterior to $x_{2}+c t, g$ and $s$ tend to zero, while for points anterior to $g$ and $s$ tend to some fixed value. This leads to the following boundary conditions:

$$
g, s \rightarrow 0 \quad \text { as } x-\left(x_{2}+c t\right) \rightarrow+\infty,
$$

$g, s$ are bounded as $x-\left(x_{2}+c t\right) \rightarrow-\infty$.

In the present paper, we solve these equations on a closed bounded interval, which we denote by $\Omega$, typically $\Omega=[-10,10]$. As an approximation to these infinite domain boundary conditions we impose zero flux boundary conditions at $x=-10$ and 10 (there is no diffusion of $s$ out of the domain). This ensures that the boundaries do not play a role in the patterning process. In the following section, we derive bounds on the parameter values according to the model assumptions.

\section{Parameter Bounds}

Constraints on the model parameter values have to be derived to ensure that the cells of a potential somite adhere at about the same time, resulting in the coordinated segmentation of a discrete somite, rather than a wave of increased adhesion travelling down the axis at constant speed $c$, which would not generate distinct somites.

The cell cycle model has different behaviours in three distinct regions along the AP axis, as illustrated in Fig. 1.

1. Cells posterior to $P_{1}\left(x>x_{1}+c t\right)$ can neither respond to nor emit a signal. For these cells, the values of $g$ and $s$ are approximately zero.

2. Cells between $P_{1}$ and $P_{2} \quad\left(x_{2}+c t\right.$ $<x \leqslant x_{1}+c t$ ) can respond to a signal but are not capable of emitting one themselves. These cells can respond to high enough values of $s$ by enhancing $G$ production greatly. As $g$ increases for these cells, $S$ production is inhibited resulting in a rapid decrease in $s$. Thus, cells in this region experience a pulse-like signal of $S$.

3. Cells at $\mathrm{P}_{2}$ emit a signal. Cells anterior to $P_{2}$ $\left(x \leqslant x_{2}+c t\right)$ can respond to the signal. These cells have a high level of $G$ which inhibits $S$ production, so their capability of signalling is substantially reduced and negligible compared with the amount of signal produced at $P_{2}$. 
To derive parameter bounds we consider each region separately after simplifying the model equations through non-dimensionalization. We non-dimensionalize the eqns (1) and (2) to allow us to give an absolute measure of the quantities involved independent of units of measurement and to reduce the number of parameters. We choose:

$$
t=\frac{\hat{t}}{\eta}, \quad x=\left(x_{1}-x_{2}\right) \hat{x}, \quad g=\frac{\hat{g}}{\eta \rho}, \quad s=\frac{\kappa \eta \rho}{\lambda} \hat{s} .
$$

In the non-dimensional variables $\hat{x}, \hat{t}, \hat{g}$ and $\hat{s}$, the distance between $x_{1}+c t$ and $x_{2}+c t$ equals 1 . We consider the process on a time-scale in which $g$ rises from near zero to its equilibrium state, in this equilibrium both $\hat{g}$ and $\hat{s}$ are $O(1)$. Substituting eqn (6) into eqns (1) and (2) and re-arranging the terms we can define the following dimensionless parameters:

$$
\begin{gathered}
\hat{\mu}=\frac{\mu \kappa}{\lambda}, \quad \hat{\gamma}=\gamma \eta^{2} \rho, \quad \hat{\kappa}=\frac{\lambda}{\eta}, \quad \hat{\varepsilon}=\varepsilon \eta \rho, \\
\hat{D}=\frac{D}{\lambda\left(x_{1}-x_{2}\right)^{2}}, \quad \hat{c}=\frac{c}{\eta\left(x_{1}-x_{2}\right)}, \quad \hat{x}_{1}=\frac{x_{1}}{x_{1}-x_{2}}, \\
\hat{x}_{2}=\frac{x_{2}}{x_{1}-x_{2}} .
\end{gathered}
$$

This yields the following non-dimensional model where the hats have been dropped for notational convenience:

$$
\begin{aligned}
& \frac{\partial g}{\partial t}=\frac{(g+\mu s)^{2}}{\gamma+g^{2}} \theta(x, t)-g, \\
& \frac{\partial s}{\partial t}=\kappa\left(\frac{\chi(x, t)}{\varepsilon+g}-s+D \frac{\partial^{2} s}{\partial x^{2}}\right),
\end{aligned}
$$

where

$$
\begin{aligned}
& \theta(x, t)=H\left(c t-x+x_{1}\right), \quad \chi(x, t)=H\left(c t-x+x_{2}\right), \\
& \quad x_{1}-x_{2}=1 .
\end{aligned}
$$

Below we indicate how the parameter bounds are found. The detailed mathematical analysis leading to these results will be reported elsewhere.

\subsection{CELLS POSTERIOR TO $P_{1}$}

For this region, $x>x_{1}+c t$ and hence the model eqns (7) and (8) become

$$
\begin{aligned}
& \frac{\partial g}{\partial t}=-g, \\
& \frac{\partial s}{\partial t}=\kappa\left(-s+D \frac{\delta^{2} s}{\delta x^{2}}\right) .
\end{aligned}
$$

The solution of these equations converges to the steady state $\left(g^{*}, s^{*}\right)=(0,0)$. In order for the signal $S$ to decay rapidly compared to $G$, we require

$$
\kappa \gg 1 .
$$

\subsection{CELLS BETWEEN $P_{1}$ AND $P_{2}$}

For this section, $x_{2}+c t<x \leqslant x_{1}+c t$. The model equations become

$$
\begin{aligned}
& \frac{\partial g}{\partial t}=\frac{(g+\mu s)^{2}}{\gamma+g^{2}}-g, \\
& \frac{\partial s}{\partial t}=\kappa\left(-s+D \frac{\delta^{2} s}{\delta x^{2}}\right) .
\end{aligned}
$$

It is required that cells between $P_{1}$ and $P_{2}$ switch from their unactivated state, with $g \approx 0$, to a potentially somitic state with a high level of $g$, in response to the signal. To obtain a non-zero stable steady state with $g$ positive, we must take

$$
\gamma<\frac{1}{4}
$$

Therefore, if $s$ is raised sufficiently, $g$ will change from approximately zero to $g_{s}=\frac{1}{2}(1+\sqrt{1-4 \gamma})$.

For the mathematical model to capture the assumption that cells posterior to $P_{1}$ do not respond to $s$ it is important to ensure that this region is not affected significantly by the diffusion of signalling molecules into it even when $s$ is low. Therefore, $g$ should remain low even if there is a constant level $s \sim 1$. After a phase plane analysis of eqn (13) it can be shown that we must choose

$$
\mu<\frac{\gamma}{4} \Rightarrow \frac{\gamma}{4 \mu}>1
$$




\subsection{CELLS ANTERIOR TO $P_{2}$}

For this region, $x \leqslant x_{2}+c t$ and hence the equations are

$$
\begin{aligned}
& \frac{\partial g}{\partial t}=\frac{(g+\mu s)^{2}}{\gamma+g^{2}}-g, \\
& \frac{\partial s}{\partial t}=\kappa\left(\frac{1}{\varepsilon+g}-s+D \frac{\partial^{2} s}{\partial x^{2}}\right) .
\end{aligned}
$$

In the section anterior to $P_{2}$, it is required that cells settle to the homogeneous steady state. It is important that $s$ rises quickly in cells passing $P_{2}$, and then decreases rapidly. Therefore, cells that experience a concentration of $S$ above some threshold should switch from a low to a high level of $G$. To ensure this, the nullcline of $s$ must lie above the local maximum at $\left(g_{M}, s_{M}\right)$ of the $g$ nullcline. If this passes below the local maximum of the $g$ nullcline, there will be three positive steady states in this region, resulting in a high steady level of $s$. From eqn (15), we can treat $\gamma$ as a small parameter, and then find approximate solutions for $g_{M}$ and $s_{M}$. These solutions permit us to determine the necessary condition for the existence of exactly one positive steady state for cells anterior to $P_{2}$, which is, $1 /\left(\varepsilon+g_{M}\right)>s_{M}$ i.e. to the lowest order in $\gamma$ :

$$
\frac{1}{\varepsilon+\gamma / 4}>\frac{\gamma}{4 \mu}
$$

In addition, for cells passing $P_{2}, g \approx 0$ and hence $s$ increases rapidly at this point. At a peak in $s, \partial^{2} s / \partial x^{2} \leqslant 0$, so $s$ cannot rise above $1 / \varepsilon$. The threshold level $s_{T}$ must therefore lie significantly below $1 / \varepsilon$, and above the local maximum $s_{M}$ of the $g$ nullcline. Hence, we take

$$
\frac{1}{\varepsilon} \gg s_{T}>s_{M} \sim \frac{\gamma}{4 \mu} \Rightarrow \frac{\gamma \varepsilon}{4 \mu} \ll 1
$$

In cells between $P_{1}$ and $P_{2}$ that have already responded to a signal, $g \sim g_{S} \sim 1$; choosing

$$
\varepsilon \ll g_{S} \sim 1
$$

ensures that $s$ is only produced in significant quantities for cells in which $g \ll 1$.

\subsection{DIFFUSION}

The posterior boundary of a somite, according to the cell cycle model, is determined by the point $P_{1}$ rather than by the diffusion of signalling molecule. If cells fail to respond to the pulse it is because they have not reached $P_{1}$ in their cell cycle, not because the signal fails to diffuse far enough. To ensure that lack of diffusion does not prevent cells becoming allocated to a single somite, we take

$$
D \gg 1 .
$$

\subsection{SUMMARY}

In summary, for eqns (7) and (8), we impose the following parameter constraints:

C1 $\kappa \gg 1$,

C2 $\gamma<\frac{1}{4}$,

C3 $\gamma / 4 \mu>1$,

C4 $1 /(\varepsilon+\gamma / 4)>\frac{\gamma}{4 \mu}$,

C5 $\gamma \varepsilon / 4 \mu \ll 1$,

C6 $\varepsilon \ll g_{S} \sim 1$, where $g_{S}=\frac{1}{2}(1+\sqrt{1-4 \gamma})$,

C7 $D \gg 1$.

\section{Spatio-Temporal Analysis}

The derivation of the parameter bounds according to the model assumptions allows us to give a qualitative description of the scenario of signal spread and cell response envisioned by the model during the formation of somites.

The cells anterior to the point $X_{0}=x_{2}+c t_{0}$ at $t=t_{0}$ have been triggered to segment, that is, they have large $G$ concentration, but the cells at $x \geqslant X_{0}$ have not responded to a signal. Initially, all cells will have low levels of signalling molecule $S$, and $g$ will be near its high steady state $g_{S}$ for $x<X_{0}$, but near zero for $x>X_{0}$. At time $t=t_{0}$, the cells at $X_{0}$ reach $P_{2}$, and $S$ is produced rapidly by these cells. The signalling molecule diffuses quickly along the embryo, causing $s$ to rise above a threshold $\left(s_{T}\right)$. 
All the cells between $P_{1}$ and $P_{2}$ receive a signal above the threshold level that enhances $G$ production, and so these cells switch to the triggered state with a high level of $G$. As $g$ increases, it inhibits $S$ production which results in a fairly rapid decrease in $s$. Thus, we have a pulse-like signal emitted from $P_{2}$.

In the cells just caudal to $P_{1}$ at $t=t_{0}$, say for $x_{1}+c t_{0}<x<X_{1}$, s will still be high enough for the cells to respond to the signal by the time they pass $P_{1}$. Hence, these cells will be specified as potentially somitic a short time after the signal is emitted. In cells posterior to $X_{1}, s$ will have fallen too low for $G$ production to be enhanced to a sufficiently high level by the time the cells are able to respond (when they pass $P_{1}$ ). These cells will have to wait until the next signal to be triggered. The result of this process is that the cells between $X_{0}$ and $X_{1}$ are triggered at approximately the same time, and hence they should go on to form a somite together. The parameter bounds derived in the previous section ensure that the signal does not persist above the threshold level for too long, otherwise cells being allocated to one somite would be triggered over a drawn out period of time.

\subsection{NUMERICAL SIMULATIONS}

The model equations pose two main difficulties: (1) the Heaviside function present in the equation for $g$ introduces a sharp discontinuity in the solution, and (2) the system is of mixed type, the $g$ equation is hyperbolic, while the $s$ equation is parabolic. A sophisticated numerical scheme was developed to solve the model equations in one spatial dimension based on the finite element method [details to be reported elsewhere].

Figure 2 shows a typical numerical simulation of eqns (7) and (8) for a set of parameter values satisfying the constraints $\mathrm{C} 1-\mathrm{C} 7$. The results show successive peaks of $s$, occurring about 20 time units apart, the first near $x=0$, the second near $x=1$, and the third near $x=2$. The process begins with a peak in $s$ at the position of $P_{2}$, quickly followed by a surge of $s$ throughout the spatial domain. The level of $s$ then decreases rapidly to a constant level. In response to this pulse-like signal, a fairly rapid increase in $g$ occurs between $P_{1}$ and $P_{2}$. Thus, we have a wave-
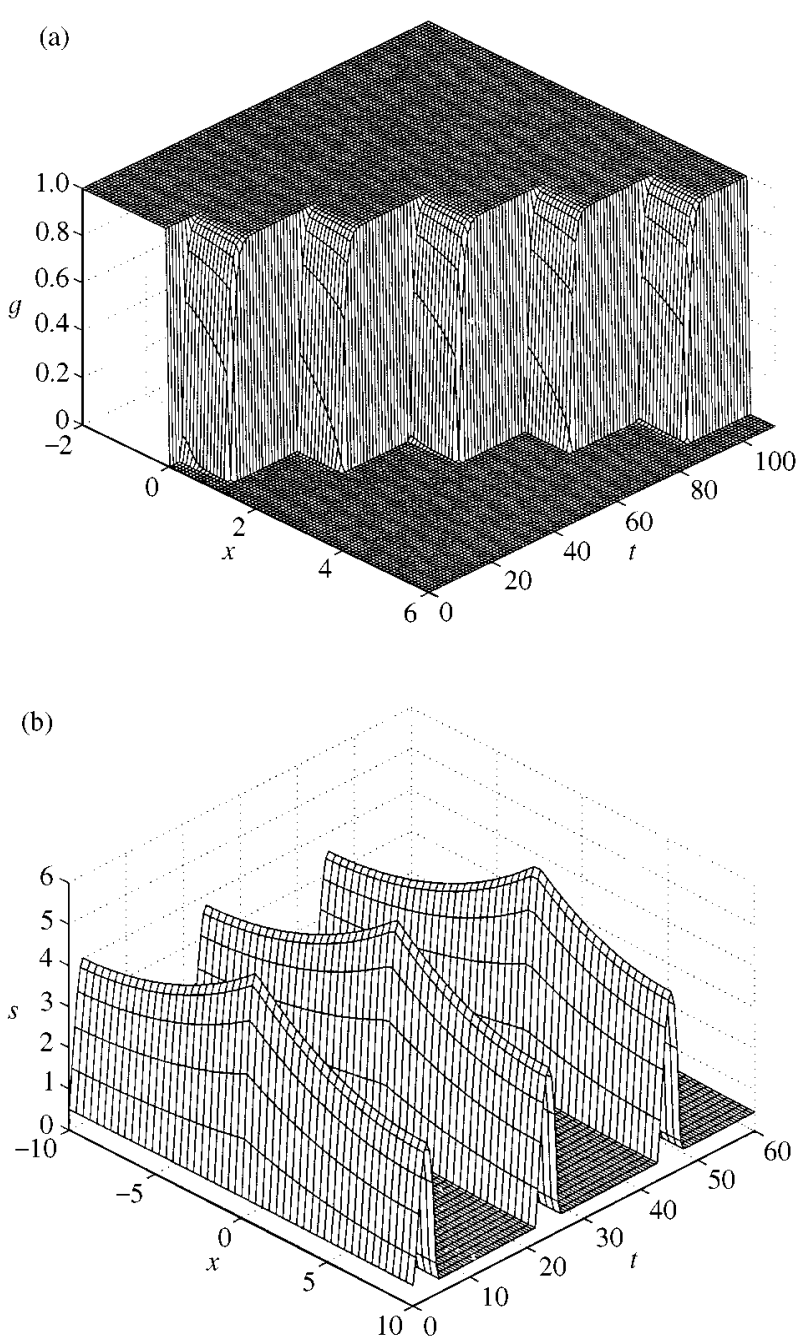

FIG. 2. Numerical solution for the model eqns (7) and (8) showing the spatio-temporal dynamics of $g(x, t)$ and $s(x, t)$. Each peak of $s$ results in an abrupt increase in $g$ which, in turn, triggers somite formation. Five "somites" are shown. (For clarity of representation only the first three $s$ peaks are shown). Parameter values are $\mu=10^{-4}, c=5 \times 10^{-2}$, $\kappa=10, \varepsilon=10^{-3}, D=100, \gamma=10^{-3}$.

front of the somitic factor $(G)$ moving down the axis in jumps, as successive groups of cells are triggered. Note that the level of $g$ rises at approximately the same time in all cells between $P_{1}$ and $P_{2}$ when a signal is emitted. According to the model, this results in the coordinated segmentation of a somite.

We have studied in detail the parameter constraints derived in Section 2. If any of these are violated the model fails to generate the required spatio-temporal pattern of somites (results not 
shown). Hence, we conjecture that these bounds are sharp.

\section{Effects of Heat Shock Experiments}

Single heat shock experiments of Primmett et al. (1988) in chick embryos resulted in multiple but discrete segmental anomalies which appeared approximately 6-7 segments after the last formed somite at the time of treatment. Up to 4 segmental anomalies could be observed in an embryo, including one small somite, one large somite or two fused somites.

Within the framework of the cell cycle model heat shock treatment of an embryo has the effect of increasing the distance between the cells at $P_{1}$ and those at $P_{2}$, since it blocks $M$ phase cells in their cell cycle. Additionally, the model assumes that the heat shock treatment does not affect the critical level of the signalling molecule, nor the strength or nature of the signal.

In Fig. 3, we present the results of the numerical solution of eqns (7) and (8) when the embryo is subjected to a heat shock. It can be seen that the second jump in $g$ is abnormally small while the third is abnormally large. The remaining jumps correspond to normal somites. In this figure, we also show the signal produced after heat shock treatment. Note that the pulse in $s$ is much sharper and higher. Figure 4 shows that the model can exhibit periodic anomalies due to heat shock.

According to the model the pattern of anomalies depends on the relation between two parameters:

1. The number of somites formed per cell cycle (l), which is approximately 6-7 somites.

2. The length of the block caused by heat shock relative to the time between $P_{1}$ and $P_{2}(\beta)$; we estimate $\beta \approx 0.6$ (equal to the time duration of heat shock divided by the time to form a somite).

The model formulation allows us to investigate this dependence and to show that, depending on the exact time and duration of heat shock, the anomalies may not be strictly periodic.

\section{Discussion}

In the present paper, we have presented and analysed a mathematical formulation of the cell
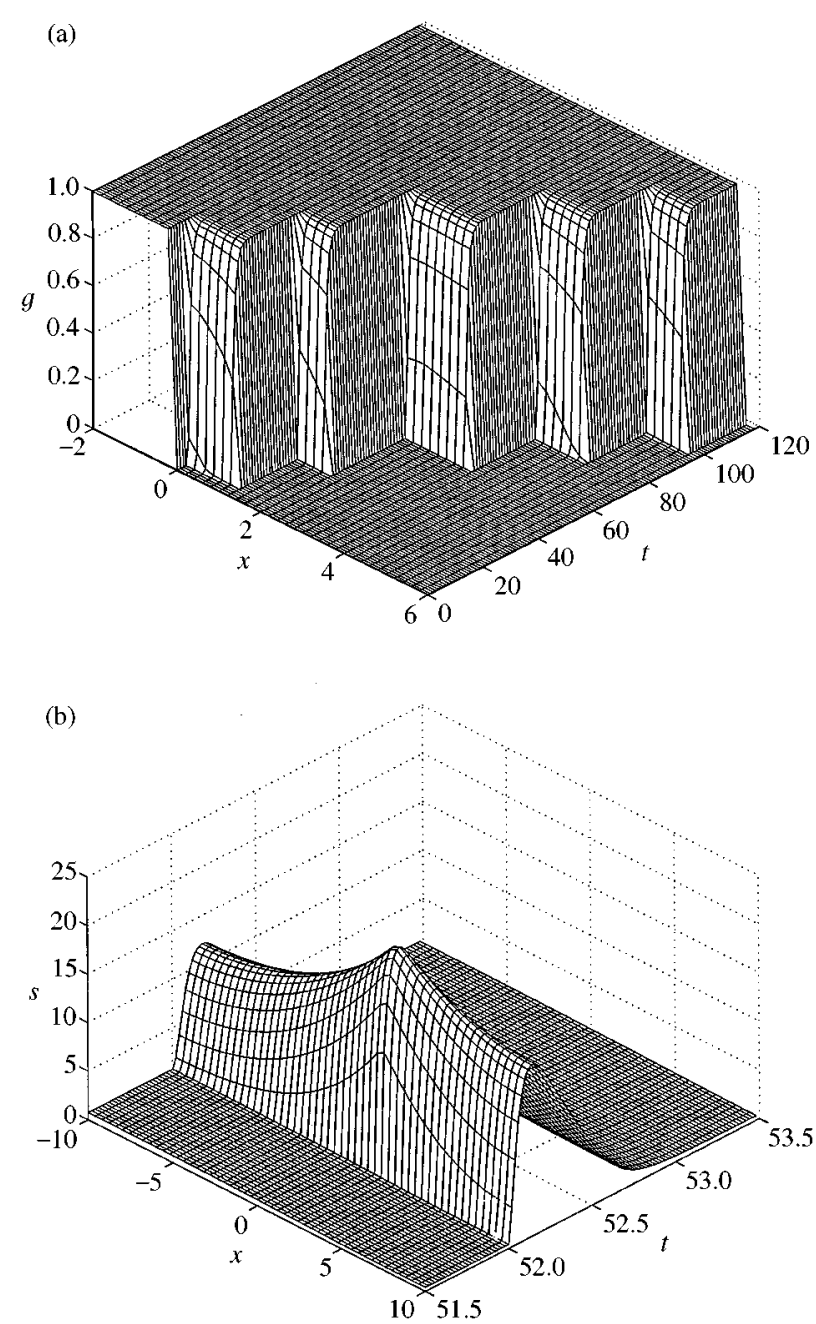

FIG. 3. Numerical solution of eqns (7) and (8) with parameter values as in Fig. 2, illustrating the effects of heat shock. In this simulation, a heat shock of duration $\beta=0.6$ (see text for details) begins to take effect at time $t=20$ resulting in one abnormally small jump (somite) in $g$ (the second one) followed by one abnormally large jump (somite). The subsequent jumps are normal (compare with Fig. 2). The signal $s$ produced after heat shock treatment is also shown (corresponding to the abnormally large somite). Note that this pulse is much higher and sharper than those in Fig. 2 which correspond to normal somite formation.

cycle model for somitogenesis proposed by Stern et al. (1988) and Primmett et al. (1989). Our analysis indicates that the cell cycle mechanism can indeed give rise to the periodic pattern of somites observed in normal embryos and also to the abnormal patterns observed after heat shock. We have incorporated cell cycle synchrony along the PSM as well as the cell-autonomous character of somite formation. Simulations could be done reversing the AP axis in a grafted explant of 

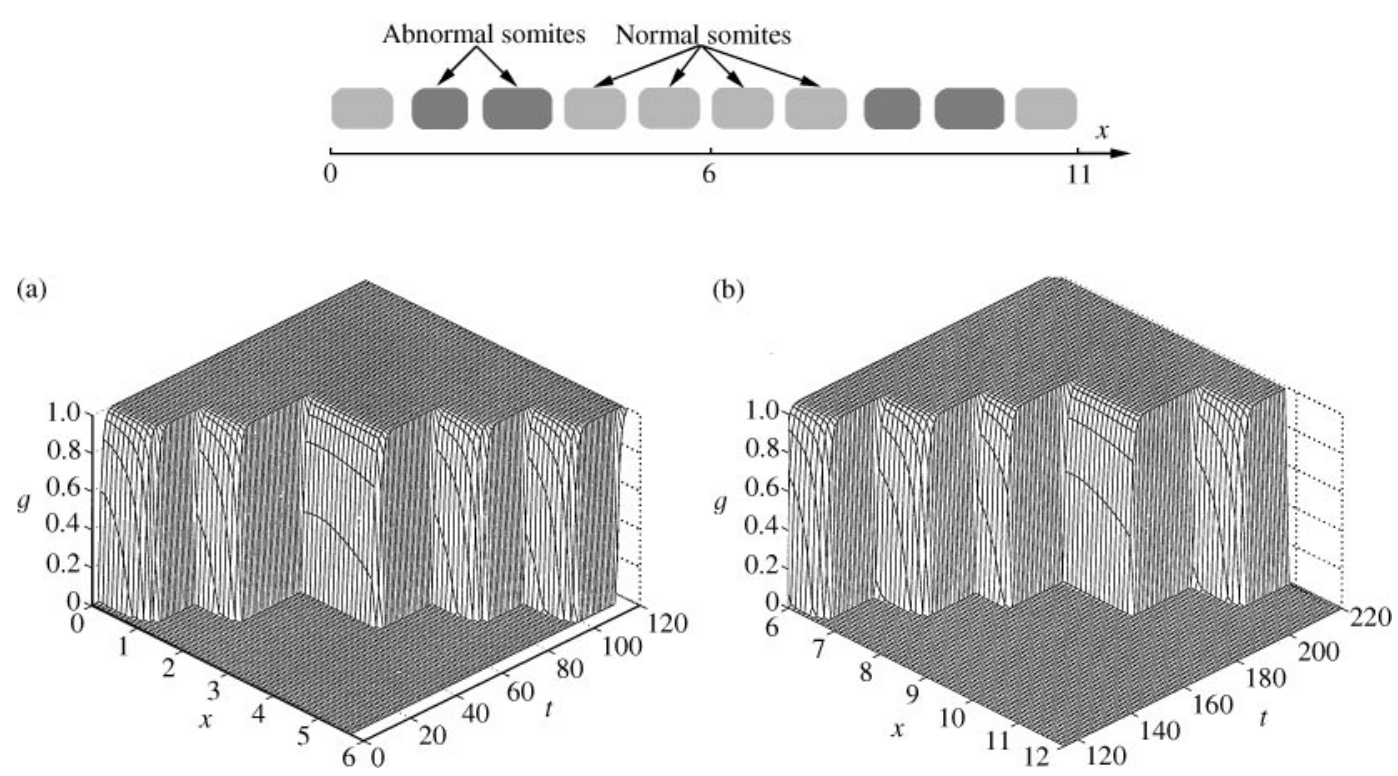

FIG. 4. Numerical solution for the model eqns (7) and (8) illustrating periodic anomalies due to heat shock treatment. Top panel is a schematic representation of the normal and abnormal somites. The heat shock treatment begins to take effect at time $t=20$ resulting in a sequence of one normal, two abnormal, six normal, two abnormal and one normal somites. Each somite of the diagram corresponds to one jump in $g$, as shown in the bottom panel (for ease of illustration we plot the dynamics of $g$ resulting in the first five somites on one diagram, and those resulting in the next five somites in the neighbouring diagram. It should be noted that this is the result of one continuous simulation). Parameter values are $\mu=10^{-4}, c=5 \times 10^{-2}$, $\kappa=10, \varepsilon=10^{-3}, \gamma=10^{-3}, D=25$. The duration of the heat shock is as in Fig. 2.

the PSM and somites will form according to their original position. The version of the model we have studied here does not incorporate cell density to study the adhesion effect, which is the focus of other work in progress.

The mathematical formulation of the cell cycle model allows us to delimit the properties that the signalling molecule must have through the parameter bounds derived in Section 3. Our numerical simulations highlight the importance of the stability of the signalling molecule $S$. If it persists for too long, then cells passing the point $P_{1}$ would be triggered and the result would be abnormally large somites. Alternatively, if $S$ is very short-lived then the somites produced would be smaller than normal. Hence, the size of the somites in this model depends crucially on the stability of $S$ which, from the mathematical formulation of the model, can be seen to depend on the diffusion coefficient and linear degradation rate of $s$.

One important point to make is that several mouse mutants reveal that although AP subdivision plays a role in determining somite bound- aries, this is NOT inextricably linked to the initial subdivision of the PSM into discrete units. For example (Kusumi et al. 1998) in most of the Notch pathway mutants (e.g. DII3/Pudgy), some sort of somites start forming despite the failure of the normal AP subdivision mechanism, but by the time that they should split into AP halves (sclerotome formation) they fall apart and regular segmentation disappears. This suggests that the hairy oscillations (this gene is part of the Notch signalling system) are more likely to be part of the AP subdivision mechanism and affect boundary formation indirectly (or late). In this paper, we restated and formulated mathematically a mechanism which regulates segmentation independently of the hairy clock and of AP subdivision. This model predicts that segmentation occurs independently from AP subdivision. This is presently being tested experimentally.

Our mathematical formulation also enables us to make experimentally testable predictions on the outcome of heat shock treatments occurring at different times and on the effects of multiple heat shock treatments. 
We have formulated the model in one dimension and have assumed that the parallel axes of the PSM are independent. Therefore, our model cannot account for the medial to lateral fused abnormalities occasionally seen in heat shock experiments. Such abnormalies also arise in certain segment polarity gene mutants (van Eeden et al., 1996, 1998). The basic mathematical model framework that we have presented in this paper could be extended to try to account for such observations.

In somitogenesis, the great experimental challenge is to understand the possible key components of the molecular clock that link the cell cycles and oscillations observed in the PSM. Further research must be carried out to study how the molecular oscillations are modulated by the cellular aspect of somitogenesis. At the same time, it is essential to understand what is the structure of the segmentation clock, how it is modulated, and what is the molecular origin of the clock.

JC would like to thank The Wellcome Trust for a Prize Studentship in Mathematical Biology. This research (DMI) is funded by a Marie Curie Fellowship as part of the Training and Mobility of Researchers (TMR) programme administered by the European Commission. SS acknowledges support from the José Gregorio Hernández Award (Academia Nacional de Medicina, Venezuela; Pembroke College, Oxford), ORS Award (Committee of Vice-Chancellors and Principals of the Universities of the United Kingdom), Programa de Confinanciamiento Institucional IVICCONICIT (CONICIT, Venezuela) and Lord Miles Senior Scholarship in Science (Pembroke College). D.J.G. would like to thank the Medical Research Council for a Career Development Fellowship.

\section{REFERENCES}

COOKE, J. (1978). Somite abnormalities caused by short heat shocks to pre-neurula stages of Xenopus laevis. J. Embryol. Exp. Morphol. 45, 283-294.

CoOKe, J. \& ZeEman, E. C. (1976). A clock and wavefront model for control of the number of repeated structures during animal morphogenesis. J. theor. Biol. 58, 455-476.

Elsdale, T., Pearson, M. \& Whitehead, M. (1976). Abnormalities in somite segmentation following heat shock to Xenopus embryos. J. Embryol. Exp. Morph. 35, 625-635.

Evrard, Y. A., Lun, Y., Aulehla, A., GAN, L. \& JOHNSON, R. L. (1998). Lunatic fringe is an essential mediator of somite segmentation and patterning. Nature 394, $377-381$.
Flint, O. P., Ede, D. A., Wilby, O. K. \& Proctor, J. (1978). Control of somite number in normal and amputated mutant mouse embryos: an experimental and a theoretical analysis. J. Embryol. Exp. Morph. 45, 189-202.

ForsberG, H., Crozet, F. \& Brown, N. A. (1998). Waves of mouse Lunatic fringe expression, in four-hour cycles at two-hour intervals, precede somite boundary formation. Curr. Biol. 8, 1027-1030.

Gossler, A. \& HrabĚ DE Angelis, M. (1998). Somitogenesis. Curr. Top. Dev. Biol. 38, 225-287.

JACOBSON, A. \& MeIER, S. (1986). Somitomeres: the primordial body segments. Somites in Developing Embryos, (Bellairs, R., Ede, D. A. \& Lash, J. W., eds), pp. 1-16. New York: Plenum Press.

Keynes, R. J. \& Stern, C. D. (1984). Segmentation in the vertebrate nervous system. Nature 310, 786-789.

Keynes, R. J. \& STERN, C. D. (1988). Mechanisms of vertebrate segmentation. Development 103, 413-429.

Kusumi, K., Sun, E. S., Kerrebrock, A. W., Bronson, R. T., ChI, D.-C., Bulotsky, M. S., Spencer, J. B., Birren, B. W., Frankel, W. N. \& Lander, E. S. (1998). The mouse pudgy mutation disrupts Delta homologue DII3 and initiation of early somite boundaries. Nature Genet. 19, 274-278.

McGrew, M. J., Dale, J. K., Fraboulet, S. \& Pourquié, O. (1998). The lunatic Fringe gene is a target of the molecular clock linked to somite segmentation in avian embryos. Curr. Biol. 8, 979-982.

McGrew, M. J. \& Pourquié, O. (1998). Somitogenesis: segmenting a vertebrate. Curr. Opin. Genet. Dev. 8, 487-493.

MEINHARDT, H. (1982). Models of Biological Pattern Formation. pp. 152-171. London: Academic Press.

MeInHARDT, H. (1986). Models of segmentation. Somites in Developing Embryos (Bellairs, R., Ede, D. A. \& Lash, J. W. eds.), pp. 179-189. New York: Plenum Press.

Müller, M., von Weizsëcker, E. \& CAMPos-OrtegA, J. A. (1996). Expression domains of a zebrafish homologue of the Drosophila pair-rule gene hairy correspond to primordia of alternating somites. Development 122, 2071-2078.

Palmeirim, I., Henrique, D., Ish-Horowicz, D. \& PourQUIÉ, O. (1997). Avian hairy gene expression identifies a molecular clock linked to vertebrate segmentation and somitogenesis. Cell 91, 639-648.

Pearson, M. \& Elsdale, T. (1979). Somitogenesis in amphibian embryos. I. Experimental evidence for an interaction between two temporal factors in the specification of somite pattern. J. Embryol. Exp. Morphol. 51, 27-50.

PolezhAEV, A. A. (1992). A mathematical model of the mechanism of vertebrate somitic segmentation. J. theor. Biol. 156, 169-181.

PolezhaEv, A. A. (1995a). Mathematical modelling of the mechanism of vertebrate somitic segmentation. J. Biol. Sys. 3, 1041-1051.

PolezHAEV, A. A. (1995b). Mathematical model of segmentation in somitogenesis in vertebrates. Biophysics 40, 583-589.

POURQUiÉ, O. (1998). Clocks regulating developmental processes. Curr. Opin. Neurobiol. 8, 665-670.

Pourquié, O. (1999). Notch around the clock. Curr. Opin. Gen. Dev. 9, 559-565.

Primmett, D. R. N., Stern, C. D. \& Keynes, R. J. (1988). Heat shock causes repeated segmental anomalies in the chick embryo. Development 104, 331-339. 
Primmett, D. R. N., Norris, W. E., Carlson, G. J., Keynes, R. J. \& Stern, C. D. (1989). Periodic segmental anomalies induced by heat shock in the chick embryo are associated with the cell cycle. Development 105, $119-130$.

Richardson, M. K., Hanken, J., Gooneratne, M. L., Pieau, C., Raynaud, A., Selwood, L. \& Wright, G. M. (1997). There is no highly conserved embryonic stage in the vertebrates: implications for current theories of evolution and development. Anat. Embryol. 196, 91-106.

Roy, M. N., Prince, V. E. \& Ho, R. K. (1999). Heat shock produces periodic somitic disturbances in the zebrafish embryo. Mech. Dev. 85, 27-34.

Schnell, S. \& Maini, P. K. (2000). Clock and induction model for somitogenesis. Dev. Dyn. 217, 415-420.

Schnell, S., PAinter, K. J., MAini, P. K. \& Othmer, H. G. (2000). Spatiotemporal pattern formation in early development: a review of primitive streak formation and somitogenesis. Mathematical Models for Biological Pattern Formation (Othmer, H. G. \& Maini, P. K., eds), pp. 11-37. New York: Springer-Verlag.

STERn, C. D. \& BELlairs, R. (1984). Mitotic activity during somite segmentation in the early chick embryo. Anat. Embryol. (Berl.), 169, 97-102.

Stern, C. D., Fraser, S. E., Keynes, R. J. \& Primmett, D. R. N. (1988). A cell lineage analysis of segmentation in the chick embryo. Development 104S, 231-244.
Stern, C. D., Hatada, Y., Selleck, M. A. J. \& Storey, K. G. (1992). Relationships between mesoderm induction and the embryonic axes in chick and frog embryos. Development Suppl., 151-156.

TAM, P. P. L. \& Trainor, P. A. (1994). Specification and segmentation of the paraxial mesoderm. Anat. Embryol. (Berl.) 189, 275-305.

van Eeden, F. J. M., Granato, M., Schach, U., Brand, M., Furutani-Seiki, M., HaffTer, P., HAmmerSCHMidT, M., HEISENBERG, C.-P., JiAnG, Y.-J., KANE, D. A., Kelsh, R. N., Mullins, M. C., Odenthal, J., Warga, R. M., Allende, M. L., Weinberg, E. S. \& NÜSSleinVOLHARD, C. (1996). Mutations affecting somite formation and patterning in the zebrafish Danio rerio. Development 123, 153-164.

van Eeden, F. J. M., Holley, S. A., Haffter, P. \& NüsSLEIN-VOLHARD, C. (1998). Zebrafish segmentation and pair-rule patterning. Devel. Genet. 23, 65-76.

VeINI, M. \& BELlairs, R. (1986). Heat shock effects in chick embryos. Somites in Developing Embryos (Bellairs, R., Ede, D. A. \& Lash, J. W., eds), pp. 135-145. New York: Plenum Press.

Wilby, O. K. \& EdE, D. A. (1975). A model for generating the pattern of cartilage skeletal elements in the embryonic chick limb. J. theor. Biol. 52, 199-217.

ZHANG, N. \& GRIDLEY, T. (1998). Defect in somite formation in lunatic fringe-deficient mice. Nature 394, 374-377. 